Check for updates

Cite this: RSC Adv., 2018, 8, 20363

Received 26th March 2018

Accepted 23rd May 2018

DOI: $10.1039 / \mathrm{c} 8 \mathrm{ra0} 2632 \mathrm{~g}$

rsc.li/rsc-advances

\section{Horseradish peroxidase-mediated synthesis of an antioxidant gallic acid-g-chitosan derivative and its preservation application in cherry tomatoes}

\begin{abstract}
Xiao Zhang, ${ }^{a}$ Hao Wu, (D) ${ }^{* a}$ Linan Zhang $^{* b}$ and Qingjie Sun (D) ${ }^{a}$
Owing to their good solubility and film-forming properties, phenolic acid-g-chitosan derivatives can be used for preservation of fruits and vegetables. However, the chemical synthesis used for the preparation of these derivatives poses a great challenge to food safety. In this study, a method involving horseradish peroxidase catalysis was used to prepare a gallic acid-g-chitosan derivative. The grafting mechanism was studied. Then, the derivative's ability to scavenge free radicals and its preserving application in cherry tomatoes were evaluated. The results indicated that the reaction for horseradish peroxidase catalysis occurred between the amino group of chitosan and the carboxyl group of gallic acid. After enzymatic grafting, the gallic acid-g-chitosan derivative possessed excellent antioxidant abilities in scavenging DPPH, hydroxyl, and superoxide anion radicals. When the derivative was used for the preservation of cherry tomatoes, the results showed that it could effectively protect the ascorbate-glutathione cycle and antioxidant enzyme system of cherry tomatoes and inhibit enzymatic browning. In addition, since this derivative delayed the postharvest senescence of cherry tomatoes, the aroma compounds remain relatively constant throughout the storage period.
\end{abstract}

\section{Introduction}

In the past few decades, the production of fresh fruit and vegetables has grown rapidly worldwide. Fruit and vegetables contain a variety of nutrients including vitamins, minerals, and antioxidants, accounting for a sizeable proportion of people's consumption. ${ }^{1}$ Among them, cherry tomatoes (Lycopersicon esculentum) are rich in carotenoids and phenolic compounds that are responsible for the high antioxidant ability of this popular fruit. ${ }^{2}$ However, cherry tomatoes are seasonal fruits, which are highly susceptible to ripening and aging during storage, thereby reducing their nutritional value. ${ }^{3}$ Consequently, it is crucial to explore strategies to prevent deterioration and extend the shelf life of cherry tomatoes.

Chitosan (CS) is a naturally occurring mucopolysaccharide with low toxicity, biodegradability, biocompatibility, and a variety of bioactivities, such as antioxidant, anticancer, antimicrobial, and enzyme-inhibitory properties. ${ }^{4}$ As a renewable resource, it has many prospective applications in many areas. However, the strong internal hydrogen bonding of CS endows its poor solubility in water. Moreover, its antioxidant capacity is mainly derived from

${ }^{a}$ College of Food Science and Engineering, Qingdao Agricultural University, Qingdao 266109, Shandong, People's Republic of China. E-mail: wuhaoqau@163.com; Tel: +8613583273291

${ }^{b}$ Marine Science and Engineering College, Qingdao Agricultural University, Qingdao 266109, Shandong, People's Republic of China. E-mail: zhanglinan1206@163.com; Tel: +8617854233253 the chelating ability of $\mathrm{N}$ atom on the amino group and the weak electron-donating ability of the amino group and hydroxyl group. Therefore, CS is considered as a secondary antioxidant, limiting its applications in food preservation. ${ }^{5}$ The modification of CS has become a new approach to develop CS derivatives while improving its solubility and imparting new properties.

Gallic acid (GA) is a natural hydroxybenzoic acid derived from nutgalls, which has a powerful ability to scavenge free radicals and inhibit oxidation chain reaction. ${ }^{6}$ However, as a sensitive antioxidant molecule, GA has poor stability and is easily degraded under high temperature, light, and other adverse conditions. Some researchers pointed out that grafting of GA onto CS molecules by chemical methods can enhance the antioxidant capacity of CS and the stability of GA. As a result of chemical grafting, the GA-CS film also showed a good preservation effect. ${ }^{5,7}$ However, the chemical synthesis has many reaction steps and may not be safe. In recent years, biocatalytic enzymes have been used for the modification and production of phenolic acid- $g$-CS derivatives. ${ }^{8}$ They are more attractive than chemical processing owing to their strong specificity and environmental friendliness. Besides, enzymes can be utilized continuously when immobilized technology is performed. ${ }^{9}$ In terms of health and safety, enzymes can also avoid harm from reactive reagents.

Among grafting reactions catalysed by enzymes, laccase, tyrosinase, and peroxidase have been widely used because of their low cost of production, relatively stable nature and a wider variety of substrates than other enzymes. ${ }^{8}$ These oxidases catalyse phenol into o-quinone intermediate, which can 
undergo two different types of reaction with CS to form either Schiff-bases $(\mathrm{C}=\mathrm{N})$ or Michael type adducts $(\mathrm{C}-\mathrm{NH})$ via covalent linkages. ${ }^{10}$ So far, laccase has been a commonly used oxidase to catalyse the grafting reaction, ${ }^{\mathbf{1 1 - 1 4}}$ while HRP was rarely used. The team of Gimeno et al. synthesized octyl gallate- $g$-CS by horseradish peroxidase (HRP), showing that the derivative achieved several good properties, such as antioxidant and antimicrobial properties as well as the adhesiveness. ${ }^{15,16}$ Considering the difference between phenolic esters and acids, it is necessary to study the synthesis of phenolic acid-g-CS derivatives by HRP and evaluate their feasibility in the preservation of fruits and vegetables benefiting from their safety and environmental friendliness.

In our previous studies, we synthesized GA-CS by laccase catalysis and found it could keep the freshness of fruits with an excellent antioxidant activity. ${ }^{\mathbf{1 3 , 1 4}}$ Thus, in this study, we attempted to graft GA onto CS via HRP-catalysed reaction to preserve cherry tomatoes. The characterization using Fouriertransform infrared spectroscopy (FT-IR), ultraviolet-visible spectroscopy (UV-vis), X-ray diffraction (XRD) and nuclear magnetic resonance spectroscopy (NMR) was conducted. Then the antioxidant activities of GA-CS synthesized by HRP on free radicals are studied, and its fresh-keeping effect on cherry tomatoes, especially some biochemical properties and endogenous enzyme activities related to the antioxidant activity of GACS was evaluated. Finally, the electronic nose was used to study the change of aroma components of cherry tomatoes during the storage.

\section{Results and discussion}

\section{Characterization of HRP-synthesized GA-CS}

CS and its grafted product were characterized by UV-vis, FT-IR, NMR and XRD technologies. As shown in Fig. 1a, no significant
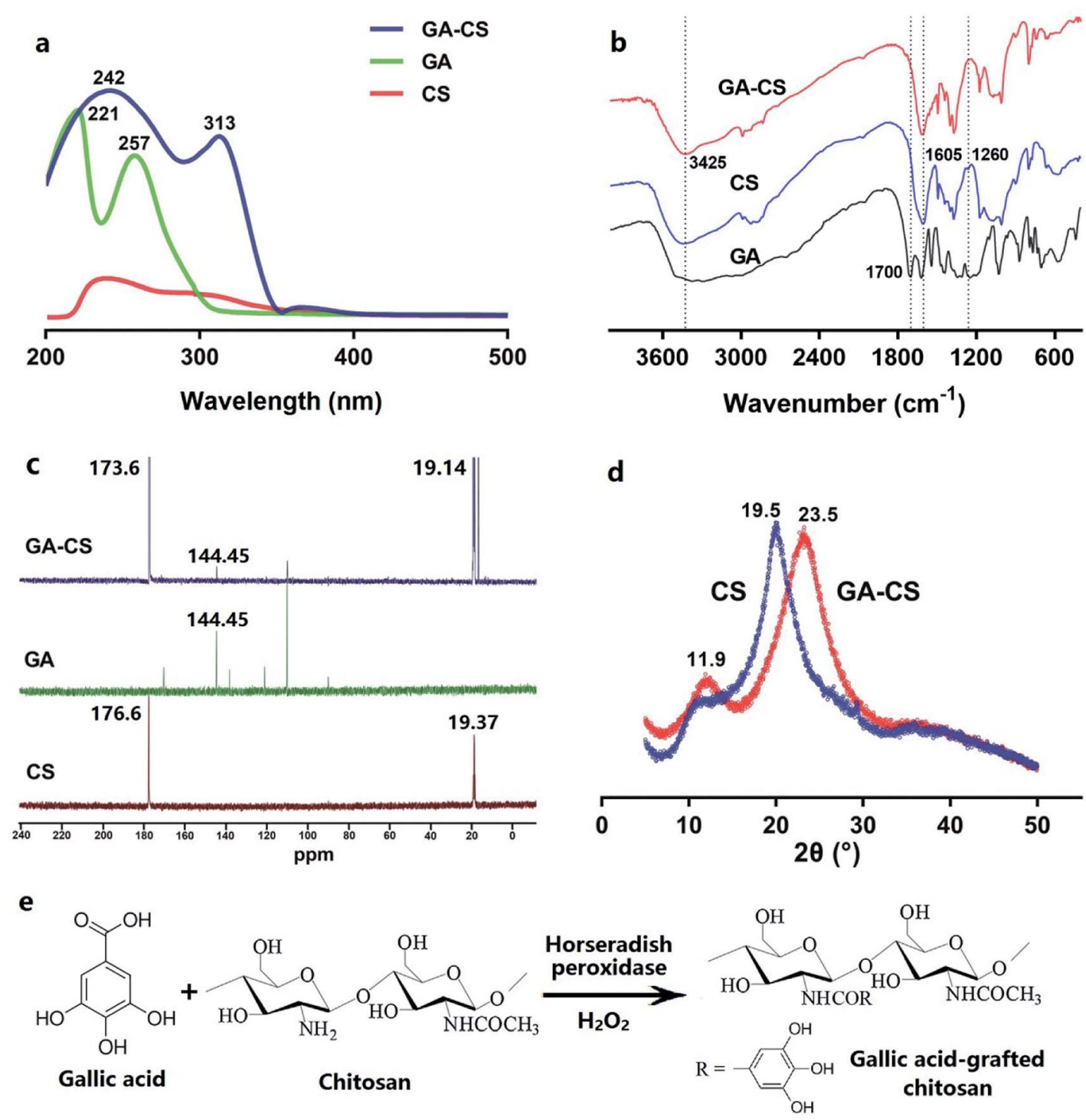

Fig. 1 UV-vis spectra (a), FT-IR spectra (b), ${ }^{13} \mathrm{C}$ NMR spectra (c), and XRD patterns (d) of CS, GA, and GA-CS. Speculated reaction pathway of horseradish peroxidase-mediated synthesis of GA-CS (e). CS represents chitosan, GA represents gallic acid, and GA-CS represents gallic acidchitosan derivative $(\mathrm{GA} / \mathrm{CS}=1: 3, \mathrm{w} / \mathrm{w})$. 
ultraviolet absorption band was observed in UV-vis spectrum of CS. In contrast, GA exhibited one band at $221 \mathrm{~nm}$ and another at $257 \mathrm{~nm}$, being attributable to the carboxy group and $\pi$-system of the benzene ring. ${ }^{11}$ After HRP incubation, the spectral features of the GA-CS were similar to GA, indicating that the chromophores in both were similar. Furthermore, the absorption band of GA-CS was broadened and shifted to 242 and $313 \mathrm{~nm}$. The observed redshifts were attributed to the less amount of energy required for the $n-\pi^{*}$ and $\pi-\pi^{*}$ transition due to the covalent linkage of GA and chitosan. ${ }^{\mathbf{1 1}}$ These results indicated that GA had been successfully grafted onto CS.

Further, the FT-IR spectra provided some structural information of CS, GA, and GA-CS. As shown in Fig. 1b, the characteristic peak of aromatic carboxyl group at $1700 \mathrm{~cm}^{-1}$ disappeared from GA after grafting onto $\mathrm{CS},{ }^{17}$ indicating that the $-\mathrm{COOH}$ of GA participated in the HRP-mediated reaction. Considering the reaction mechanism, the groups that could react with $-\mathrm{COOH}$ were $-\mathrm{OH}$ and $-\mathrm{NH}_{2}$ in $\mathrm{CS}^{8}{ }^{8}$ In Fig. $1 \mathrm{~b}$, both native $\mathrm{CS}$ and GA-CS exhibited a broadband of $3425 \mathrm{~cm}^{-1}$, which represented a stretching vibration of $-\mathrm{OH} .{ }^{11}$ However, there was no significant difference between the two peaks, suggesting that the hydroxyl group in CS was not the major site of the grafting reaction. Besides, compared to the raw $\mathrm{CS}$, peak of $\mathrm{N}-\mathrm{H}$ bending vibration $\left(1605 \mathrm{~cm}^{-1}\right)$ in GA-CS was decreased, and the characteristic amide III band (1260 $\left.\mathrm{cm}^{-1}\right)$, corresponding to a complex of $\mathrm{C}-\mathrm{N}$ stretching and $\mathrm{N}-\mathrm{H}$ in-plane bending, was disappeared from $\mathrm{CS}$ after grafting GA. ${ }^{18}$ These results illustrated that the amino group in CS reacted with carboxyl group in GA. Liu et al. indicated that the phenoxyl radicals formed by the oxidation of phenol by HRP could be further oxidized into many highly reactive intermediates. ${ }^{19}$ These intermediates could react with the free amino group of $\mathrm{CS}$ to form a covalent bond at $\mathrm{pH}<6.3,{ }^{20}$ well agreeing with the results of this study.

Solid-state ${ }^{13} \mathrm{C}$ NMR spectrometer was also used to examine the structural changes in CS before and after GA conjugation. The results were shown in Fig. 1c. The peaks at $176.6 \mathrm{ppm}(\mathrm{C}=$ O) and $19.37 \mathrm{ppm}\left(-\mathrm{CH}_{3}\right)$ were observed in $\mathrm{CS}$, corresponding to the carbonyl and methyl groups of $\mathrm{N}$-acetylglucosamine, respectively. ${ }^{21}$ In the spectrum of GA-CS, besides the carbonyl (173.6 ppm) and methyl groups (19.14 ppm) from CS, a new signal at $144.45 \mathrm{ppm}$ was observed, representing the $\mathrm{C}=\mathrm{C}$ of gallate group from GA. ${ }^{21}$ In addition, the increased peak intensity at $173.6 \mathrm{ppm}$ was presumably due to the introduction of $\mathrm{C}=\mathrm{O}$ between $\mathrm{CS}$ and carboxyl groups of GA. These results further confirmed the conjugation of GA on CS chains.

Moreover, the crystallographic structure of CS, GA, and GA-CS were determined by XRD approach. As shown in Fig. 1d, the diffraction pattern of CS showed a characteristic peak at $19.5^{\circ}$, corresponding to crystal form $\mathrm{II}^{22}$ while GA-CS showed two broader peaks at $23.5^{\circ}$ and $11.9^{\circ}$, confirming the successful conjugation of the gallate group onto the CS. Moreover, XRD results revealed that the introduction of GA onto CS caused a decrease in crystallinity of the grafted copolymers. This result suggested that intermolecular hydrogen bindings in GA-CS reduced compared to $\mathrm{CS} .{ }^{21}$ Therefore, the XRD data concluded that the solubility of GA-CS was better than CS, which was consistent with the results from Pasanphan and Chirachanchai. ${ }^{23}$
Finally, the possible reaction pathway for HRP-catalysed grafting of GA onto CS in the presence of $\mathrm{H}_{2} \mathrm{O}_{2}$ was shown in Fig. 1e. In general, GA was oxidized by HRP into intermediates with higher activity, which could be covalently bound to the amino groups of CS to generate GA-CS.

\section{Antioxidant capacity of GA-CS}

It has been reported that tomato fruit ripening is closely related to its oxidative status. ${ }^{24}$ Thus, the antioxidant property of GA$\mathrm{CS}$, which is important in the postharvest storage of cherry tomatoes, is evaluated by the ability to scavenge the free radicals, including 1,1-diphenyl-2-picrylhydrazyl (DPPH), hydroxyl radicals $\left(\mathrm{OH}^{*}\right)$, and superoxide anion radical $\left(\mathrm{O}_{2}{ }^{-}\right)$.

DPPH is a free radical that accepts an electron or hydrogen radical and eventually becomes a stable molecule. As shown in Fig. 2a, CS had a weak inhibitory activity on the DPPH with an
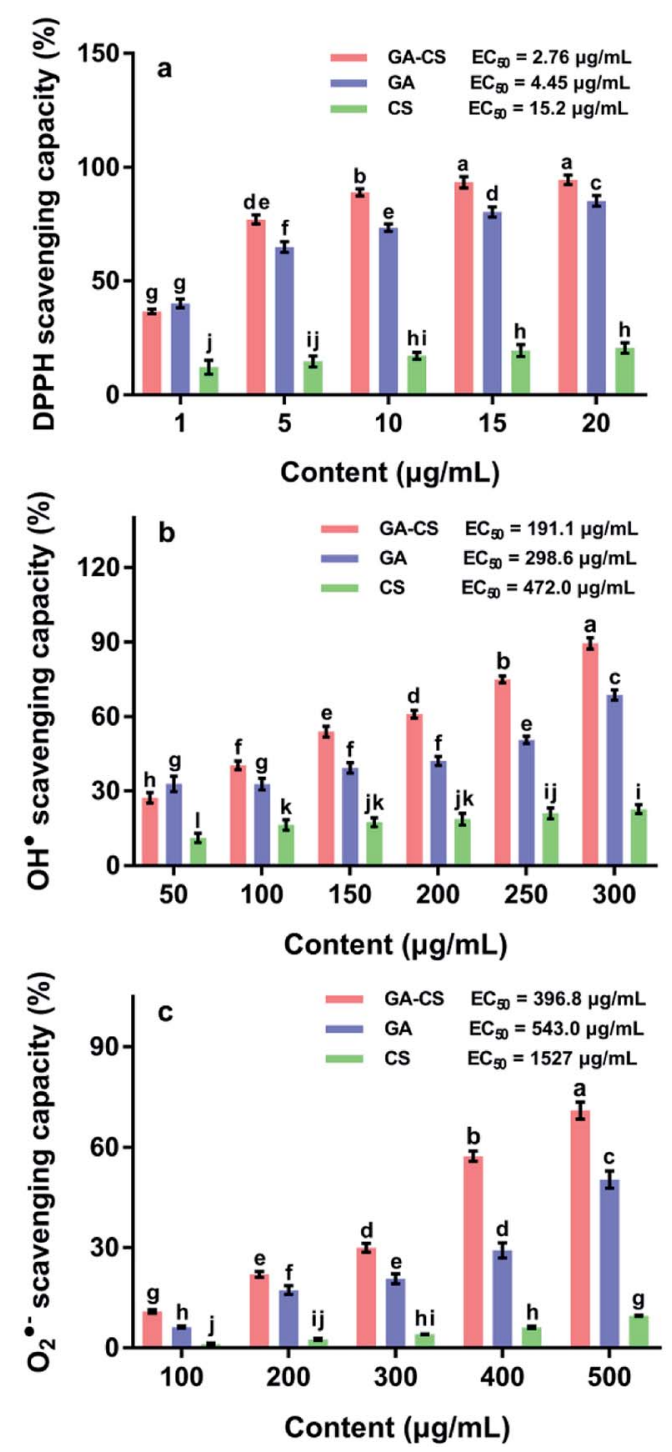

Fig. $2 \mathrm{DPPH}$ (a), $\mathrm{OH}^{*}$ (b), and $\mathrm{O}_{2}{ }^{\cdot-}$ (c) scavenging capacity of CS, GA and GA-CS. CS represents chitosan, GA represents gallic acid, and GA-CS represents gallic acid-chitosan derivative (GA/CS $=1: 3, \mathrm{w} / \mathrm{w}$ ). The same letters indicate no significant difference $(p>0.05)$. 
$\mathrm{EC}_{50}$ value of $15.2 \mu \mathrm{g} \mathrm{mL}^{-1}$. Its antioxidant activity might be related to the reaction of free radicals with the hydroxyl group at the C- 6 position through $\mathrm{H}$-abstraction, and the reaction of free radicals with the amino group at the $\mathrm{C}-2$ position to form stable macromolecule radicals. ${ }^{25}$ After grafting GA, CS derivatives had the strongest ability to scavenge DPPH except at the low concentration of $1 \mu \mathrm{g} \mathrm{mL}{ }^{-1}(p<0.05)$. The $\mathrm{EC}_{50}$ of GA-CS and GA were 2.76 and $4.45 \mu \mathrm{g} \mathrm{mL}{ }^{-1}$, respectively. Thus, the DPPH scavenging capacity of GA-CS was improved by introducing GA into CS and seemed totally dependent on the amount of GA introduced.

Approaching the next step, the $\mathrm{OH}^{*}$ scavenging capacity of GA-CS, GA and CS was examined via the Fenton reaction. As shown in Fig. 2b, all test samples scavenged $\mathrm{OH}^{*}$ in a dosedependent manner. The $\mathrm{EC}_{50}$ values of GA-CS, GA and CS for were $191.1,298.6$, and $472.0 \mu \mathrm{g} \mathrm{mL}^{-1}$, respectively. This result indicated that the CS showed the lowest antioxidant potential against $\mathrm{OH}^{*}$ whereas GA-CS was the strongest. This antioxidant activity appeared to be dependent on the phenolic hydroxyl groups in GA, which was the main active group capable of scavenging $\mathrm{OH}^{*}{ }^{26}$

Further, antioxidant efficiency of GA-CS was also evaluated by $\mathrm{O}_{2}{ }^{-}$- scavenging assay. As shown in Fig. 2c, CS showed only a radical scavenging rate of $9.5 \%$ at $500 \mu \mathrm{g} \mathrm{mL} \mathrm{m}^{-1}$, whereas the scavenging capacity of GA-CS continued to increase, eventually reaching $71 \%$. The ability of GA to scavenge $\mathrm{O}_{2}{ }^{-}-$was also increased with an increasing concentration, and the maximum rate could be observed at 50.3\% . Compared their $\mathrm{EC}_{50}$, GA-CS showed a minimum, corresponding to the strongest scavenging ability. This result was similar to the result in Fig. $2 \mathrm{a}$ and $\mathrm{b}$. However, the $\mathrm{O}_{2}{ }^{-}{ }^{-}$scavenging efficiency of GA-CS was not as high as DPPH and $\mathrm{OH}^{*}$, which might be due to different scavenging mechanisms of different radicals.

\section{Effects of GA-CS on ascorbic acid-glutathione cycle of cherry tomato}

As discussed above, introduction of GA into CS could improve its antioxidant capacity. Then, during the post-harvest storage period, some physiological indicators of cherry tomatoes closely related to the antioxidant property of GA-CS were further evaluated.

Commonly, ascorbic acid (ASA) and glutathione (GSH) constitute the ASA-GSH cycle, which is an important part of the non-enzymatic antioxidant systems in plants. ${ }^{27}$ The ASA-GSH cycle is responsible for the removal of $\mathrm{H}_{2} \mathrm{O}_{2}$, which can stimulate the increase of enzyme activity in the cycle and increase the scavenging activity of reactive oxygen species (ROS) under moderate stress conditions. ${ }^{27}$ However, ASA and GSH are usually oxidized and degraded with the ripening of postharvest fruits. As shown in Fig. 3a, ASA level of all cherry tomatoes continued to decline throughout storage. After 10 days, GA-CS treatment showed a higher VC level $(14.72 \mathrm{mg} / 100 \mathrm{~g})$ than other treatments. The above result indicated that HRP-mediated GA-CS derivative could effectively inhibit the oxidation of ASA in cherry tomatoes.

Additionally, the GSH content decreased at the early stage of storage, then increased slightly (Fig. 3b). This might be because
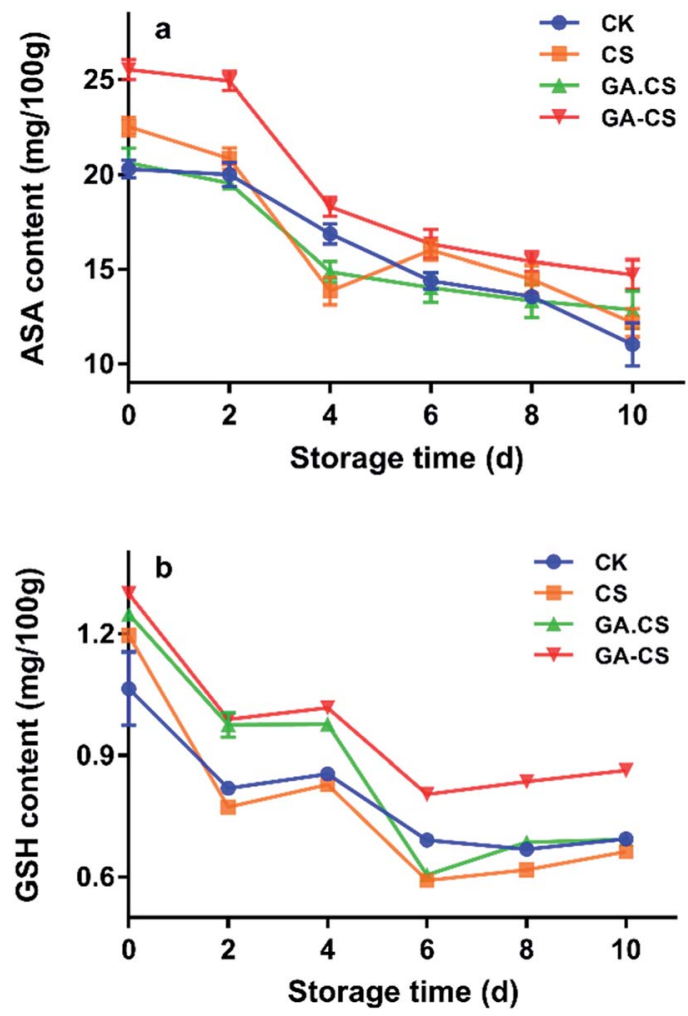

Fig. 3 Changes in ASA (a) and GSH (b) of cherry tomatoes during storage. CS represents chitosan, GA represents gallic acid, GA.CS represents the simple mixing of $\mathrm{GA}$ and $\mathrm{CS}(\mathrm{GA} / \mathrm{CS}=1: 3, \mathrm{w} / \mathrm{w})$, and $\mathrm{GA}-\mathrm{CS}$ represents gallic acid-chitosan derivative (GA/CS $=1: 3, \mathrm{w} / \mathrm{w})$. ASA is ascorbic acid, GSH is reduced glutathione.

cherry tomatoes consumed ASA during storage, resulting in a decrease in GSH content in the ASA-GSH cycle. In the late stage of storage, glutathione reductase reduced oxidized glutathione (GSSH) to GSH due to increased oxidative stress caused by senescence. ${ }^{28}$ After GA-CS treatment of cherry tomatoes, the GSH content was up to $0.864 \mathrm{mg} / 100 \mathrm{~g}$ after storage for 10 days, which was significantly higher than that of the control group ( $p$ $<0.05$ ). This might be because GA-CS had a good antioxidant capacity, which in turn maintain the high activity of related enzymes to protect GSH in cherry tomatoes.

In summary, GA-CS prepared in this study alleviated the degradation of ASA and GSH in cherry tomatoes during storage, which to a certain degree ensured that the ASA-GSH cycle proceeded effectively.

\section{Effects of GA-CS on enzymatic browning of cherry tomato}

Like ASA, polyphenols are common antioxidants in cherry tomatoes. They not only bring the enjoyment of colour and taste to humans but also bring health benefits. ${ }^{29}$ Phenolics in postharvest cherry tomatoes are highly unstable and undergo a various change that deeply affects their taste and nutritional quality. For instance, postharvest handling and transportation induce the subcellular decompartmentalization that stimulates the leakage of phenolic substances from vacuoles, allowing them to contact with polyphenols oxidase (PPO) and peroxidase 
(POD).$^{30}$ Then, polyphenols are degraded and oxidized to quinines, followed by brown pigments.

Fig. 4a showed the change in polyphenols in cherry tomatoes during 10 days under different treatments. During the whole storage period, the polyphenols showed a slowly decreasing trend, and the GA-CS group experienced the slowest decline, indicating that GA-CS could inhibit the oxidation of polyphenols. This result might be due to the high ASA content in cherry tomatoes that were treated by GA-CS, and its excellent reducibility prevented the degradation of phenolic compounds. ${ }^{31}$ Furthermore, PPO activity was consistently lower in the GA-CS treated cherry tomatoes than in the other three groups (Fig. 4b). After 10 days, a significant difference in PPO activity was observed between GA-CS group $(0.0668 \mathrm{U}$ $\left.\mathrm{g}^{-1} \min ^{-1}\right)$ and other three groups $(p<0.05)$. The result showed
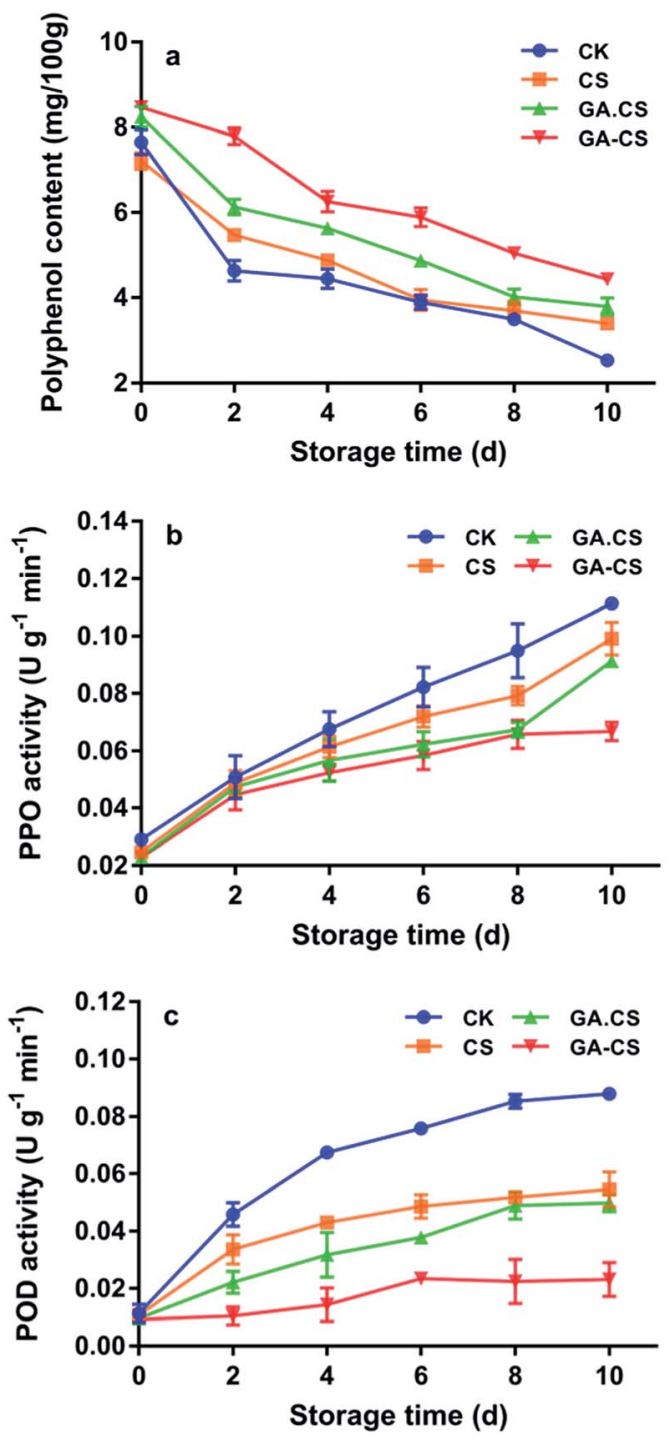

Fig. 4 Polyphenol content (a), PPO activity (b), and POD activity (c) of cherry tomatoes during storage. CS represents chitosan, GA represents gallic acid, GA.CS represents the simple mixing of GA and CS $(G A / C S=1: 3, w / w)$, and GA-CS represents gallic acid-chitosan derivative (GA/CS $=1: 3, \mathrm{w} / \mathrm{w}$ ). PPO is polyphenol oxidase, POD is peroxidase. that combination of CS and GA delayed the increase of PPO activity and inhibited enzymatic browning of cherry tomatoes during storage. The antioxidant GA protected the polyphenols in cherry tomatoes and the film-forming property of CS provided an oxygen barrier function. ${ }^{32}$

Just as PPO, peroxidase (POD) is also involved in browning reactions and producing melanin compounds. It catalyses the decomposition of $\mathrm{H}_{2} \mathrm{O}_{2}$ in the presence of a hydrogen donor, such as hydroxycinnamic derivatives and flavans. ${ }^{33}$ As shown in Fig. 4c, POD activity of GA-CS group showed almost unchanged from the initial state within the first 4 days and reached 0.0231 $\mathrm{U} \mathrm{g}^{-1} \mathrm{~min}^{-1}$ on the $10^{\text {th }}$ day, while the CK group reached 0.0458 $\mathrm{U} \mathrm{g}^{-1} \mathrm{~min}^{-1}$ on the second day. This result indicated that GACS effectively inhibit the PPO activity. In summary, the antioxidant GA-CS was able to maintain the phenolic content and control the enzymatic browning of cherry tomatoes after harvesting.

\section{Effects of GA-CS on antioxidant enzymes of cherry tomato}

As discussed above, owing to its excellent antioxidant capacity, GA-CS synthesized by HRP can protect the ASA-GSH cycle and inhibit enzymatic browning of cherry tomatoes during storage. Then, the effects of GA-CS treatment on activities of some antioxidant enzymes of cherry tomatoes were studied.

In addition to non-enzymatic antioxidants, there are also some enzymatic antioxidants in plants, such as superoxide dismutase (SOD) and catalase (CAT). ${ }^{34}$ These antioxidant enzymes can remove ROS before their participation in oxidation reactions and alleviate the gradual increase of oxidative stress caused by senescence of fruits and vegetables. ${ }^{35}$ Generally, in plant cells, the first line of defence was SOD, which converted $\mathrm{O}_{2}{ }^{--}$to $\mathrm{H}_{2} \mathrm{O}_{2}$. As shown in Fig. $5 \mathrm{a}$, the SOD activity in the control group on the second and tenth days was 3.03 and $16.79 \mathrm{U}$ $\mathrm{mL}^{-1} \mathrm{~min}^{-1}$, respectively. The decrease of SOD activity in the later period of storage indicated the ability to scavenge ROS was weakened. However, after 10 days, the SOD activities of the CS, GA.CS, and GA-CS groups were 5.34, 5.74, and $6.42 \mathrm{U}$ $\mathrm{mL}^{-1} \mathrm{~min}^{-1}$ respectively, indicating that these treatments could effectively protect the activity of SOD.

Moreover, when $\mathrm{H}_{2} \mathrm{O}_{2}$ is overproduced from $\mathrm{O}_{2}{ }^{\cdot-}$, it is further decomposed by CAT, which converts $\mathrm{H}_{2} \mathrm{O}_{2}$ to water. As shown in Fig. 5b, similar to SOD, the CAT activity in the control group also increased first and then decreased. The maximum value was $0.0683 \mathrm{U} \mathrm{mL}^{-1} \mathrm{~min}^{-1}$ on the fourth day, showing that CAT activity was activated by oxidative stress early in senescence. By the tenth day, CAT activity of cherry tomatoes was minimized to $0.0546 \mathrm{U} \mathrm{mL}^{-1} \mathrm{~min}^{-1}$. However, the change in CAT activity of cherry tomatoes coated by GA-CS during storage fluctuated, and a maximum of $0.0853 \mathrm{U} \mathrm{mL}^{-1} \mathrm{~min}^{-1}$ was retained. The above result showed that GA-CS could protect the antioxidant enzyme system, enhance the ability to remove ROS, improve anti-aging ability, and extend cherry tomatoes' shelf life.

\section{Effects of GA-CS on aroma compounds of cherry tomato}

In general, every fruit has its own characteristic aroma because of the presence of unique monoterpenes, esters, organic acids, 

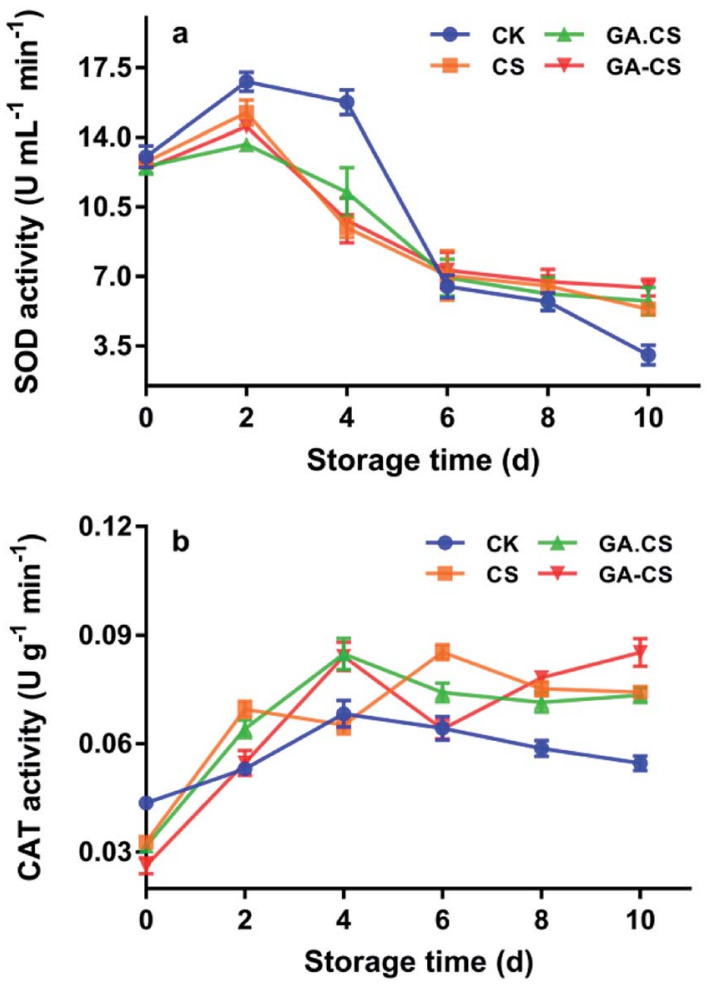

Fig. 5 SOD (a) and CAT (b) activities of cherry tomatoes during storage. CS represents chitosan, GA represents gallic acid, GA.CS represents the simple mixing of GA and $C S(G A / C S=1: 3, w / w)$, and GA-CS represents gallic acid-chitosan derivative (GA/CS $=1: 3, \mathrm{w} / \mathrm{w})$. SOD is superoxide dismutase, CAT is catalase.

aldehydes, ketones. With the advancing ripening, production of volatile components usually increases. ${ }^{36}$ Thus, aroma changes of different coated cherry tomatoes were assessed by principal component analysis during storage.

As shown in Fig. 6, although the trend was not an irregular distribution along the first and second principal components, the fruity substances at different storage time were concentrated. They had no overlapping area and were easily distinguishable from each other, which was consistent with another study using electronic nose to evaluate the shelf life of tomato storage.$^{37}$ Besides, the first principal components (axis- $x$ ) of CK, CS, GA. CS and GA-CS treatment were $93.75 \%, 94.71 \%, 95.47 \%$, and $98.20 \%$, respectively. The second principal component (axis-y) was $5.87 \%, 4.65 \%, 4.35 \%$, and $1.41 \%$, respectively. Therefore, the first principal component contributed a lot to the overall aroma, while the contribution of the second principal component was negligible.

As shown in Fig. 6a, the aroma of the control group was slightly different in the first four days. From the $6^{\text {th }}$ day to the $10^{\text {th }}$ day, the aroma was significantly different, indicating that the physiological activity of the control group during the later period of storage was active, and the aroma changed more. As shown in Fig. 6b and c, the response distance of CS and GA. CS group during storage was large, showing that the aroma components of the cherry tomatoes varied significantly after the two treatments. As shown in Fig. 6d, in addition to the eighth day, the first principal component of GA-CS group changed little during the entire storage period, and the second principal component changed greatly. However, since the second principal component could be neglected in the overall characteristic aroma, this result also showed that the aroma components in cherry tomatoes treated by GA-CS were stable, reflecting the good fresh-keeping effect of GA-CS on cherry tomatoes. Therefore, compared with other treatments, GA-CS can maintain the stability of cherry tomato aroma, well agreeing with the results of other physical and chemical indicators.

\section{Experimental}

\section{Materials}

CS $\left(M_{\mathrm{w}} 500 \mathrm{kDa}\right)$ with a deacetylation degree of $90.8 \%$ was purchased from Qingdao YunZhou Biotechnology Co., Ltd., China. Before use, CS dissolved in a $2 \%(\mathrm{w} / \mathrm{v})$ acetic acid was purified by precipitation using $\mathrm{NaOH}$. The precipitate was washed several times with a great amount of deionized water until the $\mathrm{pH}$ reached 7.0. GA was obtained from Tianjin Kermel Chemical Reagent Co., Ltd. (Tianjin, China). HRP (EC1.11.1.17, $206 \mathrm{U} \mathrm{mg}^{-1}$ ) was purchased from Beijing Solarbio Science \& Technology Co., Ltd. (Beijing, China). Fresh cherry tomatoes (Lycopersicon esculentum) were purchased from a local supermarket (Qingdao, China). All other reagents were of grade and were used as received without further purification.

\section{Preparation of GA-CS}

CS solution $(1 \%, \mathrm{w} / \mathrm{v})$ was prepared by agitating CS flakes in an acetic acid/sodium acetate buffer solution (pH 4.5) at ambient temperature overnight. Then GA was added to CS solution with a GA/CS ratio of $(1: 3, \mathrm{w} / \mathrm{w})$. After homogeneous distribution, $\operatorname{HRP}\left(4 \mathrm{U} \mathrm{mg}^{-1}\right)$ was slowly added whilst stirring, $1 \mathrm{~mL}$ of $30 \%$ $\mathrm{H}_{2} \mathrm{O}_{2}$ was added to the reaction solution, reacting for $5 \mathrm{~h}$ at $25{ }^{\circ} \mathrm{C}$. The unreacted ingredients and the by-products were removed by dialyzing with 3500 molecular weight cut-off dialysis tubing against distilled water for $72 \mathrm{~h}$. The precipitate obtained was centrifuged and freeze-dried to obtain the GA-CS.

\section{UV-vis, FT-IR, NMR, and XRD analysis}

UV-vis spectra of GA-CS sample were recorded using a UV-vis 2450 spectrophotometer (Shimadzu Corporation, Japan) in a range of $200-500 \mathrm{~nm}$. FT-IR spectra were recorded on a Nicolet Nexus 670 infrared spectrophotometer (Thermo Fisher Scientific Inc., USA) with 32 scans at a resolution of $2 \mathrm{~cm}^{-1}$ in a frequency range of $4000-400 \mathrm{~cm}^{-1}$. Solid state ${ }^{13} \mathrm{C}$ NMR test was performed on a Bruker UltraShield 600 PLUS NMR spectrometer (Bruker, Germany). Freeze-dried hydrogels were used and operated at a recording frequency of $100.63 \mathrm{MHz}$ and spinning rate of $8 \mathrm{kHz}$. XRD patterns were obtained with X-ray diffractometer (Bruker AXS, Germany) using $\mathrm{Cu}$ as $\mathrm{K}_{\alpha}$ radiation, and the Bragg's angle $2 \theta$ was scanned from 5 to $50^{\circ}$ at the rate of $0.1^{\circ} \mathrm{min}^{-1}$.

\section{Determination of antioxidant activity}

The DPPH scavenging capacity of the GA-CS was quantified according to the method of Mishra et al. ${ }^{38}$ Briefly, the derivatives with different concentrations were mixed with and without DPPH 

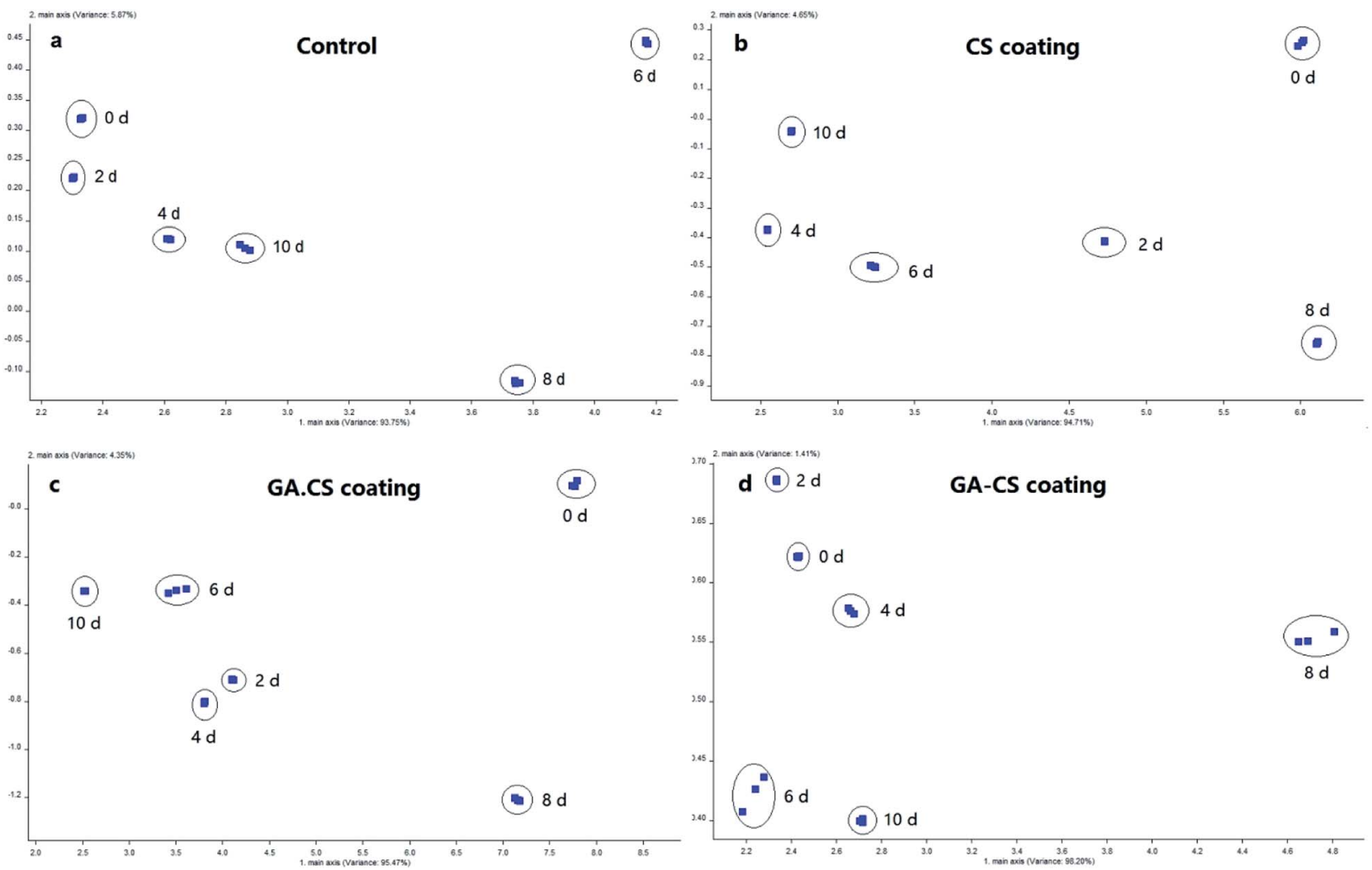

Fig. 6 Electronic nose data - principal component analysis results of cherry tomatoes treated with control (a), CS (b), GA·CS (c), GA-CS (d). GA.CS represents the simple mixing of $\mathrm{GA}$ and $\mathrm{CS}(\mathrm{GA} / \mathrm{CS}=1: 3, \mathrm{w} / \mathrm{w})$, and $\mathrm{GA}-\mathrm{CS}$ represents gallic acid-chitosan derivative $(\mathrm{GA} / \mathrm{CS}=1: 3, \mathrm{w} / \mathrm{w})$.

solution $(0.1 \mathrm{mM})$, then the mixtures were incubated at $25{ }^{\circ} \mathrm{C}$ for $30 \mathrm{~min}$. The DPPH scavenging capacity of the coating solutions absorbance of the mixtures was determined at $517 \mathrm{~nm}$. Scavenging effect of GA-CS was calculated by the following equation,

$$
\text { Scavenging capacity } \%=\frac{1-\left(A_{\mathrm{s}}-A_{\mathrm{i}}\right)}{A_{0}} \times 100 \%
$$

where $A_{0}$ is the absorbance of the control, $A_{\mathrm{s}}$ is the absorbance of the sample, and $A_{\mathrm{i}}$ is the absorbance of the sample under identical condition as $A_{\mathrm{s}}$ with ethanol instead of DPPH solution.

The $\mathrm{OH}^{*}$ scavenging assay was performed based on Smirnoff and Cumbes. ${ }^{39} 1 \mathrm{~mL}$ of $\mathrm{FeSO}_{4}(6 \mathrm{mM})$, sample solutions with different concentration, $1 \mathrm{~mL}$ of $\mathrm{H}_{2} \mathrm{O}_{2}(6 \mathrm{mM})$ and $1 \mathrm{~mL}$ of salicylic acid solution was mixed and incubated at $37^{\circ} \mathrm{C}$ for $30 \mathrm{~min}$. The scavenging activity of $\mathrm{OH}^{*}$ was evaluated by the following equation,

$$
\text { Scavenging capacity } \%=\frac{1-\left(A_{\mathrm{s}}-A_{\mathrm{i}}\right)}{A_{0}} \times 100 \%
$$

where $A_{0}$ was the absorbance of the distilled water at $510 \mathrm{~nm}, A_{\mathrm{i}}$ was the absorbance of the distilled water replaced by salicylic acid, and $A_{\mathrm{s}}$ was the absorbance of the sample.

The $\mathrm{O}_{2}{ }^{--}$scavenging capacity of samples were examined by a pyrogallol autoxidation system with minor modification. ${ }^{40}$ Briefly, $3 \mathrm{~mL}$ of Tris- $\mathrm{HCl}$ buffer solution ( $50 \mathrm{mM}$, pH 8.2) was incubated in a water bath at $25{ }^{\circ} \mathrm{C}$ for $20 \mathrm{~min}$, and then reacted with $10 \mu \mathrm{L}$ of pyrogallol solution $(3 \mathrm{mM})$ for $3 \mathrm{~min}$, at last $50 \mu \mathrm{L}$ ascorbic acid was added. The absorbance at $420 \mathrm{~nm}\left(A_{0}\right)$ was measured $30 \mathrm{~s}$ later, indicating the speed of pyrogallol autoxidation. The $A_{1}$ autoxidation speed was obtained using the above method with the addition of test compounds $(0.2 \mathrm{~mL})$ into the Tris-HCl buffer. A blank control of reagent was obtained as $A_{2}$. The scavenging activity of $\mathrm{O}_{2}{ }^{--}$was evaluated by the following equation,

Scavenging capacity $\%=\frac{1-\left(A_{1}-A_{2}\right)}{A_{0}} \times 100 \%$

\section{Pretreatment of cherry tomatoes}

Cherry tomatoes were washed and randomly divided into 4 groups, and consequently soaked into different coating solutions for $2 \mathrm{~min}$, including $10 \mathrm{mg} \mathrm{mL}^{-1}$ of CS dissolved in acetic acid solution, $250 \mathrm{mg}$ GA and $750 \mathrm{mg}$ CS dissolved in $100 \mathrm{~mL}$ acetic acid solution (GA.CS), and $10 \mathrm{mg} \mathrm{mL}^{-1} \mathrm{GA}-\mathrm{CS}$ dissolved in deionized water. After draining, the cherry tomatoes were then packed in PE plastic bag and stored at $15{ }^{\circ} \mathrm{C}$ (the temperature used in display cabinets of fresh fruits and vegetables in a supermarket) for determination of ASA, GSH, polyphenol, and some endogenous enzyme analysis. These parameters were measured every two days.

\section{ASA content}

The content of ASA was determined using the 2, 6-dichloroindophenol method. ${ }^{41} 10 \mathrm{~g}$ of cherry tomato pulp was vortex 
mixed with $100 \mathrm{~mL}$ oxalic acid $\left(20 \mathrm{~g} \mathrm{~L}^{-1}\right)$. The mixture was then centrifuged at $8000 \mathrm{~g}$ for $10 \mathrm{~min}$ to obtain the supernatant. $10 \mathrm{~mL}$ of the supernatant was titrated with 2,6-dichlorophenolindophenol to a reddish colour. The titration volume was recorded when the colour was stable for at least $30 \mathrm{~s}$. Oxalic acid solution $\left(20 \mathrm{~g} \mathrm{~L}^{-1}\right)$ was used as the blank control with titration performed as described above. The ASA content (mg/ $100 \mathrm{~g}$ ) was calculated as follows:

$$
\mathrm{ASA}=\frac{V \times\left(V_{1}-V_{0}\right) \times c}{V_{\mathrm{s}} \times m} \times 100
$$

where $V_{1}$ was the titration volume for the sample $(\mathrm{mL}), V_{0}$ was the titration volume for the blank control (mL), $V$ was the total supernatant volume $(\mathrm{mL}), c$ was the ASA content titrated by $1 \mathrm{~mL}$ of 2,6-dichlorophenol, $V_{\mathrm{s}}$ was the volume of the sample $(\mathrm{mL})$, and $m$ was the sample mass $(\mathrm{g})$.

\section{Polyphenol content}

$5 \mathrm{~g}$ of cherry tomatoes was homogenized and mixed with $20 \mathrm{~mL}$ of $60 \%(\mathrm{v} / \mathrm{v})$ ethanol. After extraction for $2 \mathrm{~h}$ and filtration, the volume was set to $500 \mathrm{~mL}$. The content of polyphenol was determined by the Folin-Ciocalteu method with some modification. ${ }^{42}$ The reaction mixture was composed of $1 \mathrm{~mL}$ of cherry tomatoes extracts, $1 \mathrm{~mL}$ of Folin-Ciocalteu reagent, and $3 \mathrm{~mL}$ of $20 \%(\mathrm{w} / \mathrm{w}) \mathrm{NaCO}_{3}$ solution. After incubation for $2 \mathrm{~h}$ at $25{ }^{\circ} \mathrm{C}$ in darkness, the absorbance of samples was measured at $760 \mathrm{~nm}$. The polyphenol content was expressed in terms of GA equivalents based on a standard curve.

\section{GSH content}

The content of GSH was measured based on 2-vinylpyridine method..$^{43}$ In detail, the reaction was initiated by adding 1 unit of glutathione reductase and monitored the increase in absorbance at $412 \mathrm{~nm}$. For measuring oxidized glutathione (GSSG), $20 \mu \mathrm{L}$ of the extract was mixed with $200 \mu \mathrm{L}$ of phosphate buffer $(0.5 \mathrm{M}, \mathrm{pH}=7.5)$ and $4 \mu \mathrm{L}$ of 2 -vinylpyridine, and the mixture was incubated at $25{ }^{\circ} \mathrm{C}$ for $30 \mathrm{~min}$ to remove $\mathrm{GSH}$ by derivatization. GSSG was assayed by the same way as for total GSH previously. GSH content was determined by subtracting the value for GSSG from the total GSH content.

\section{Endogenous enzyme activity}

For determination of endogenous enzyme activity in cherry tomatoes, samples $(0.1 \mathrm{~g})$ were ground in a chilled mortar with $1 \%(\mathrm{w} / \mathrm{v})$ polyvinylpolypyrrolidone and then homogenized with $1.2 \mathrm{~mL}$ of $50 \mathrm{mM}$ potassium phosphate buffer (PB) (pH 7.8) containing $1 \mathrm{mM}$ EDTA-Na $\mathrm{N}_{2}$ and $0.3 \%$ (w/v) Triton X-100. Each homogenate was centrifuged at $13000 \mathrm{~g}$ for $20 \mathrm{~min}$ at $4{ }^{\circ} \mathrm{C}$ and the supernatant was used for the following assays. Activities of peroxidase (POD), catalase (CAT) and superoxide dismutase (SOD) were all measured according to Wang et al. ${ }^{\mathbf{4 4}}$ Activity of polyphenol oxidase (PPO) was measured based on Chisari et al. ${ }^{45}$

\section{Analysis of aroma compounds}

The aroma ingredients were analyzed by a PEN3 portable E-nose instrument (Win Muster Airsense Analytics Inc., Germany). According to methods previously reported, ${ }^{\mathbf{4 6}}$ each sample was weighed $40.0 \mathrm{~g}$ in a sample vial before being sealed. After equilibrating for $90 \mathrm{~min}$ at $25{ }^{\circ} \mathrm{C}$, the hermetic vial was pierced by a Luer lock needle connected to a $3 \mathrm{~mm}$ Teflon tubing by using headspace air intake. The flow rate of sample introduction and

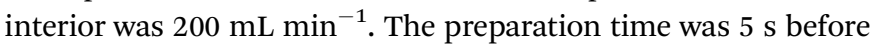
E-nose measurement began, and the measurement process lasted for $60 \mathrm{~s}$, during which the absorbed gases were measured each second. The E-nose system was automatically zero cleared for $60 \mathrm{~s}$ and then standardized for $300 \mathrm{~s}$. Afterward, the next headspace sampling started repetitively until all samples were examined. Each sample was measured for five repeats. The relatively stable signal at 55-57 s was extracted as feature data for subsequent principal component analysis.

\section{Statistical analysis}

Data were expressed as the mean \pm standard deviation of three separate experiments. All statistical analysis was conducted using SPSS 19.0 software (SPSS Inc., USA). The Shapiro-Wilk test was used to assess the normality of data and the Levene test was used to check the homoscedasticity. The difference between the heating treatments was evaluated using the one-way analysis of variance (ANOVA) and Duncan's multiple comparisons. ${ }^{47}$

\section{Conclusions}

In this study, the HRP-catalysed method was used to prepare GACS. The reaction mechanism was explored by UV-vis, FT-IR, NMR, and XRD technologies. Then, antioxidant abilities of GA-CS were studied, and fresh-keeping effects of GA-CS on cherry tomatoes were evaluated. The results showed that the formation of GA-CS might begin with the highly active intermediates derived from GA oxidized by HRP. Then, covalent bond was formed between amino groups of CS and carboxyl group of GA. After enzymatic grafting, GA-CS showed the excellent antioxidant capacity and could scavenge $\mathrm{DPPH}, \mathrm{OH}^{*}$, and $\mathrm{O}_{2}{ }^{-}{ }^{-}$. When applying in the preservation of cherry tomato, GA-CS could effectively protect the ASA-GSH cycle, maintain the activity of intracellular antioxidant enzymes, and inhibit the enzymatic browning. In addition, the aroma components of cherry tomatoes were relatively constant throughout the storage period because the postharvest aging of cherry tomatoes was delayed.

\section{Conflicts of interest}

There are no conflicts to declare.

\section{Acknowledgements}

This study was funded by National Natural Science Foundation of China (31401549), Special Funds for Taishan Scholars Project of Shandong Province, Shandong Modern Vegetable Industrial Technology System (SDAIT-05-21), Agriculture Scientific and 
Technological Innovation Project of Shandong Academy of Agriculture Sciences (CXGC2016B10) and Talents of High Level Scientific Research Foundation of Qingdao Agriculture University (631207).

\section{References}

1 A. Septembre-Malaterre, F. Remize and P. Poucheret, Food Res. Int., 2018, 104, 86-99.

2 O. Masetti, A. Ciampa, L. Nisini, P. Sequi and M. T. Dell'Abate, Food Res. Int., 2017, 100, 623-630.

3 S. Wu, M. Lu and S. Wang, Food Chem., 2016, 199, 296-300. 4 W. Xia, P. Liu, J. Zhang and J. Chen, Food Hydrocolloids, 2011, 25, 170-179.

5 B. Schreiber, J. J. Bozell, D. G. Hayes and S. Zivanovic, Food Hydrocolloids, 2013, 33, 207-214.

6 S. Roidoung, K. D. Dolan and M. Siddiq, Food Chem., 2016, 210, 422-427.

7 P. Guo, J. D. Anderson, J. J. Bozell and S. Zivanovic, Carbohydr. Polym., 2016, 140, 171-180.

8 J. Liu, H. Pu, S. Liu, J. Kan and C. Jin, Carbohydr. Polym., 2017, 174, 999-1017.

9 O. Vittorio, M. Cojoc, M. Curcio, U. G. Spizzirri, S. Hampel, F. P. Nicoletta, F. Iemma, A. Dubrovska, M. Kavallaris and G. Cirillo, Macromol. Chem. Phys., 2016, 217, 1488-1492.

10 F. Hollmann and I. W. Arends, Polymers, 2012, 4, 759-793.

11 M. Božič, S. Gorgieva and V. Kokol, Carbohydr. Polym., 2012, 87, 2388-2398.

12 A. Aljawish, I. Chevalot, J. Jasniewski, C. Paris, J. Scher and L. Muniglia, Food Chem., 2014, 145, 1046-1054.

13 H. Wu, Y. Wang, T. Zhen, D. Luo, X. Zhang, S. Yang and C. Wang, Food Sci., 2017, 38, 227-232.

$14 \mathrm{H}$. Wu, T. Zhen, C. Chen, Y. Wang, D. Luo, X. Zhang and C. Wang, Transations of the Chinese Society of Agricultural Engineering, 2017, 33, 285-292.

15 L. Itzincab-Mejía, A. López-Luna, M. Gimeno, K. Shirai and E. Bárzana, Int. J. Food Sci. Technol., 2013, 48, 2034-2041.

16 L. Zavaleta-Avejar, E. Bosquez-Molina, M. Gimeno, J. P. Pérez-Orozco and K. Shirai, Food Hydrocolloids, 2014, 39, 113-119.

17 W. Pasanphan, G. R. Buettner and S. Chirachanchai, J. Appl. Polym. Sci., 2008, 109, 38-46.

18 A. Sionkowska, B. Kaczmarek, K. Lewandowska, S. Grabska, M. Pokrywczyńska, T. Kloskowski and T. Drewa, Int. J. Biol. Macromol., 2016, 89, 442-448.

19 N. Liu, S. Ni, A. J. Ragauskas, X. Meng, N. Hao and Y. Fu, J. Biotechnol., 2018, 269, 8-15.

20 A. Aljawish, I. Chevalot, J. Jasniewski, J. Scher and L. Muniglia, J. Mol. Catal. B: Enzym., 2015, 112, 25-39.

21 J. Liu, J. F. Lu, J. Kan and C. H. Jin, Int. J. Biol. Macromol., 2013, 62, 321-329.
22 R. J. Samuels, Solid state characterization of the structure of chitosan films, J. Polym. Sci., Polym. Chem. Ed., 1981, 19, 1081-1105.

23 W. Pasanphan and S. Chirachanchai, Carbohydr. Polym., 2008, 72, 169-177.

24 A. Jimenez, G. Creissen, B. Kular, J. Firmin, S. Robinson, M. Verhoeyen and P. Mullineaux, Planta, 2002, 214, 751-758.

25 W. Xie, P. Xu and Q. Liu, Bioorg. Med. Chem. Lett., 2001, 11, 1699-1701.

26 J. W. Chen, Z. Q. Zhu, T. X. Hu and D. Y. Zhu, Acta Pharmacol. Sin., 2002, 23, 667-672.

27 A. Mirto, F. Iannuzzi, P. Carillo, L. F. Ciarmiello, P. Woodrow and A. Fuggi, Food Chem., 2018, 240, 559-566.

28 S. S. Gill and N. Tuteja, Plant Physiol. Biochem., 2010, 48, 909930.

29 A. Crozier, I. B. Jaganath and M. N. Clifford, Nat. Prod. Rep., 2009, 26, 1001-1043.

30 P. M. Toivonen and D. A. Brummell, Postharvest Biol. Technol., 2008, 48, 1-14.

31 E. Cocci, P. Rocculi, S. Romani and M. D. Rosa, Postharvest Biol. Technol., 2006, 39, 265-271.

32 G. Kerch, Trends Food Sci. Technol., 2015, 46, 159-166.

33 L. Vamos-Vigyazo, Crit. Rev. Food Sci. Nutr., 1981, 15, 49-127.

34 O. Blokhina, E. Virolainen and K. V. Fagerstedt, Ann. Bot., 2003, 91, 179-194.

35 B. Halliwell, Plant Physiol., 2006, 141, 312-322.

36 F. R. Thewes, A. Brackmann, R. de Oliveira Anese, E. S. Bronzatto, E. E. Schultz and R. Wagner, Postharvest Biol. Technol., 2017, 127, 1-13.

37 V. Giovenzana, R. Beghi, S. Buratti, R. Civelli and R. Guidetti, Talanta, 2014, 120, 368-375.

38 K. Mishra, H. Ojha and N. K. Chaudhury, Food Chem., 2012, 130, 1036-1043.

39 N. Smirnoff and Q. J. Cumbes, Phytochemistry, 1989, 28, 1057-1060.

40 A. J. Sfahlan, A. Mahmoodzadeh, A. Hasanzadeh, R. Heidari and R. Jamei, Food Chem., 2009, 115, 529-533.

41 X. Liu, J. Ren, Y. Zhu, W. Han, H. Xuan and L. Ge, Colloids Surf., A, 2016, 502, 102-106.

42 M. Musci and S. Yao, Int. J. Food Sci. Nutr., 2017, 68, 913-918. 43 O. W. Griffith, Anal. Biochem., 1980, 106, 207-212.

44 L. Y. Wang, J. L. Liu, W. X. Wang and Y. Sun, Photosynthetica, 2016, 54, 19-27.

45 M. Chisari, R. N. Barbagallo and G. Spagna, J. Agric. Food Chem., 2007, 55, 3469-3476.

46 Q. Li, X. Yu, L. Xu and J. M. Gao, Food Chem., 2017, 221, 1113-1119.

47 D. Granato, V. M. de Araújo Calado and B. Jarvis, Food Res. Int., 2014, 55, 137-149. 